\title{
Training-Induced Gene Expression Plasticity in Cardiac Function and Neural Regulation for Ultra-Trail Runners
}

\author{
María Maqueda $^{1,2}$, Emma Roca ${ }^{3}$, Daniel Brotons ${ }^{4}$, J. Manuel Soria ${ }^{5}$, Alexandre Perera ${ }^{1,2}$ \\ ${ }^{1}$ Dep. ESAII, Biomedical Engineering Research Centre, Technical University of Catalonia (UPC), \\ Barcelona, Spain \\ ${ }^{2}$ Biomedical Research Networking Center in Bioengineering, Biomaterials and Nanomedicine \\ (CIBER-BBN), Spain \\ ${ }^{3}$ Facultad Medicina, Universitat de Girona, Spain \\ ${ }^{4}$ Àrea Medicina de l’Esport, Consell Català de l’Esport, Barcelona, Spain \\ ${ }^{5}$ Unitat de Genomica de Malalties Complexes, Institut de Recerca de l'Hospital de la Santa Creu i \\ Sant Pau, Barcelona, Spain
}

\begin{abstract}
This study aims to assess the gene regulatory response from a group of 16 athletes and to observe the plasticity induced by their training regime on the gene expression response after their participation in an $82 \mathrm{~km}$ race. Blood samples for differential gene expression (DGE) were collected before and after this effort from two groups of runners with different training regimes: elite and active. Analyses only focused on genes annotated as related to cardiac function (CF) and neural regulation (NR) from the KEGG PATHWAY Database. Thus, 13 pathways were considered accounting for a total of 629 genes.

Training regime modulated the response to exercise based on a list of 18 ranked genes with significant DGE for elite runners while remained statistically insignificant for active athletes. UQCR11, COX7C and COX4I1 genes, related to mitochondrial respiratory chain, were downregulated which may indicate mitochondrial function impairment in cardiac muscle. Increased expression levels were obtained for PIK3R2, PLCG2, IRAK3 genes from the positive signaling cascades of neurotrophins pathway, which may reveal an improved heart rate control thanks to a better cardiac sympathetic innervation.
\end{abstract}

\section{Introduction}

Ultra-trail races are an ideal sport to investigate a physiological response to an endurance exercise given its significant intensity and prolonged duration [1]. The study of heart rate variability is a powerful and an extended method for better understanding the cardiac function and its regulation through the autonomic nervous system [2]. Most experiments have focused on examining the effects of exercise training versus sedentary subjects [3] or in previously sedentary individuals [4]. The training intensity, duration and type have already been related with specific genetic regulation activity [5].

The aim of this work is to analyze the gene expression plasticity, induced by distinct training regimes followed by runners, in the physiological response to an ultra-trail participation.

\section{Materials and methods}

\subsection{Experimental design}

A total of 16 runners were recruited and classified into two different training regime groups based on their weekly exercise hours: (i) Active group from three to 10 hours and (ii) Elite group: more than 10 hours.

Eight male and two female formed the active group with mean age of $38.7 \pm 4.7$ years, while three male and three female belonged to the elite group (38.0 \pm 1.4 years). Characteristics of the runners are summarized in Table 1.

All individuals were experienced athletes who volunteered to participate in this experiment, which was deployed on the Cavalls del Vent 4th edition from 2012 (Cadí-Moixeró Natural Park, Catalonia, Spain). The crossing took place at altitudes of between 900 meters to 2510 meters with a total positive slope of 5200 meters approximately. Ultra-trail distance was $82 \mathrm{~km}$. The active group could run a mean distance of $37.3 \pm 14.1 \mathrm{~km}$ and the elite group $60.3 \pm 23.9 \mathrm{~km}$. Adverse weather conditions caused that only three out of 16 participants were able to complete the race. 
Table 1. Runner characteristics participating in the experiment.

\begin{tabular}{lllll}
\hline Id & $\begin{array}{l}\text { Training } \\
\text { level }\end{array}$ & Gender & $\begin{array}{l}\text { Dist }^{1} \\
{[\mathrm{~km}]}\end{array}$ & $\begin{array}{l}\text { Age } \\
\text { [Years] }\end{array}$ \\
\hline $1^{2}$ & Elite & Female & 82 & 36 \\
15 & Elite & Female & 82 & 38 \\
2 & Elite & Male & 42 & 40 \\
3 & Elite & Female & 82 & 39 \\
7 & Elite & Male & 33 & 38 \\
$9^{2}$ & Elite & Male & 41 & 37 \\
163 & Active & Male & 50 & 45 \\
164 & Active & Male & 50 & 42 \\
197 & Active & Male & 25 & 37 \\
256 & Active & Male & 50 & 37 \\
577 & Active & Male & 25 & 42 \\
735 & Active & Male & 50 & 40 \\
$775^{2}$ & Active & Male & 28 & 28 \\
805 & Active & Male & 50 & 36 \\
$838^{2}$ & Active & Female & 31 & 42 \\
957 & Active & Female & 14 & 38 \\
\hline
\end{tabular}

${ }^{1}$ Dist: Distance completed by the runner.

${ }^{2}$ Only pre-race expression levels are available.

\subsection{Blood sampling, RNA isolation and transcriptome analysis}

Venous blood samples were drawn from the antecubital vein at rest in a sitting position and collected into PAXgene Blood RNA Tubes according to the manufacturer's protocol (PreAnalytiX GmbH/QIAGEN, Switzerland/US). Samples were obtained from each subject in previous periods before the race and after completing their participation in the ultra-trail. They were stored at $-80^{\circ} \mathrm{C}$ and properly identified until their assay in Hospital de la Santa Creu I Sant Pau (HSCiSP) (Barcelona, Spain).

Total RNA was isolated using the PAXgene Blood RNA kit (PreAnalytiX GmbH/QIAGEN, Switzerland/US). The concentration of the extracted RNA was measured spectrophotometrically (Nanodrop 1000/ Thermo Fisher Scientific, Wilmington, US). Expression levels were randomly checked by real time PCR performed on IL-1 $\beta$ and CD141 genes.

For expression profiling, RNA was amplified and biotinylated using the Ambion TotalPrep RNA Amplification kit (Life Technologies, Carlsbad US) in order to be measured through hybridization onto HuGene2.0st microarrays (Affymetrix Inc., California US). HSCiSP laboratory carried out the hybridization, washing, staining, scanning and grid alignment processes in order to generate the corresponding CEL files.

\subsection{Bioinformatic data analysis}

A selection of biomarkers candidates to regulate and control the cardiovascular system was made based on a prior selection of related biological pathways from KEGG PATHWAY database [6]. With this purpose, a total of 13 pathways (see Table 2) belonging to circulatory system (CS) and nervous system (NS), from organismal systems category, were chosen.

Table 2. List of pathways related to CF and NR from KEGG PATHWAYS Database - circulatory system (CS) and nervous system (NS) category.

\begin{tabular}{ll}
\hline $\begin{array}{l}\text { KEGG } \\
\text { Pathway id }\end{array}$ & Pathway name \\
\hline hsa04260 & $\begin{array}{l}\text { Cardiac muscle contraction (CS) } \\
\text { hsa04261 }\end{array}$ \\
& $\begin{array}{l}\text { Adrenergic signaling in cardiomyocytes } \\
\text { (CS) }\end{array}$ \\
hsa04270 & $\begin{array}{l}\text { Vascular smooth muscle contraction } \\
\text { (CS) }\end{array}$ \\
hsa04720 & Long-term potentiation (NS) \\
hsa04721 & Synaptic vesicle cycle (NS) \\
hsa04722 & Neurotrophin signaling pathway (NS) \\
hsa04723 & $\begin{array}{l}\text { Retrograde endocannabinoid signaling } \\
\text { (NS) }\end{array}$ \\
hsa04724 & Glutamatergic synapse (NS) \\
hsa04725 & Cholinergic synapse (NS) \\
hsa04726 & Serotonergic synapse (NS) \\
hsa04727 & GABAergic synapse (NS) \\
hsa04728 & Dopaminergic synapse (NS) \\
hsa04730 & Long-term depression (NS) \\
\hline
\end{tabular}

Available CEL files (28 in total) were analyzed with the R Software for Statistical Computing (v3.2.0) [7] and BioConductor v3.1 [8]. Raw fluorescence intensity values were background corrected, quantile normalized and summarized with Robust Multichip Average through package oligo v1.32.0 [9]. An ExpressionSet object was obtained with expression level values for 53,617 transcript clusters per each athlete in the study. Quality control was performed over this object in order to detect possible outliers. A list of 629 unique biomarkers was selected based on the former pathway selection. Only this subset was considered for further assessment.

A non-supervised filtering based on overall intensity and variability was applied to discard non-informative genes resulting in 77 genes. Based on the design of the experiment, three parameters (Gender: $G$, Training level: $T$ and time - pre or post-race: $P P$ ) were identified to have an impact on gene expression. Thus, a linear regression model was fit to each biomarker expression value $\left(g_{k}\right)$ by controlling for those variables and an interaction factor between the latter as indicated below (1). Package limma 
Table 3. Ranked genes with differential expression levels in elite vs active runners as a response to ultra-trail participation sorted by obtained adj. p-value (FDR).

\begin{tabular}{llllll}
\hline Transcript & Gene & Regulation & adj. p-val & logFC & KEGG Pathways Id \\
\hline 16827366 & ATP6V0D1 & Up & $2.52 \mathrm{E}-06$ & 1.05 & hsa04721 \\
16690566 & SORT1 & Up & $1.26 \mathrm{E}-05$ & 1.42 & hsa04722 \\
16821614 & COX4I1 & Down & $4.80 \mathrm{E}-05$ & -0.89 & hsa04260 \\
16859763 & PIK3R2 & Up & $1.66 \mathrm{E}-04$ & 0.86 & hsa04261;hsa04722;hsa04725 \\
16668286 & GNAI3 & Up & $2.04 \mathrm{E}-04$ & 0.86 & hsa04261;hsa04723;hsa04724;hsa04725;hsa04726; \\
& & & & & hsa04727;hsa04728;hsa04730 \\
16753670 & IRAK3 & Up & $3.26 \mathrm{E}-04$ & 1.35 & hsa04722 \\
16837391 & KCNJ2 & Up & $4.13 \mathrm{E}-04$ & 1.13 & hsa04725 \\
17007543 & ITPR3 & Down & $5.62 \mathrm{E}-04$ & -0.82 & hsa04270;hsa04720;hsa04723;hsa04724;hsa04725; \\
& & & & & hsa04726;hsa04728;hsa04730 \\
17095111 & GNAQ & Up & $6.02 \mathrm{E}-04$ & 0.77 & hsa04261;hsa04270;hsa04720;hsa04723;hsa04724; \\
& & & & & hsa04725;hsa04726;hsa04728;hsa04730 \\
16944096 & ATP6V1A & Up & $1.07 \mathrm{E}-03$ & 0.83 & hsa04721 \\
16866718 & UQCR11 & Down & $1.40 \mathrm{E}-03$ & -0.61 & hsa04260 \\
16821326 & PLCG2 & Up & $1.77 \mathrm{E}-03$ & 0.83 & hsa04722 \\
16986983 & COX7C & Down & $2.65 \mathrm{E}-03$ & -0.87 & hsa04260 \\
17002846 & DUSP1 & Up & $5.12 \mathrm{E}-03$ & 0.73 & hsa04726 \\
16866974 & GNG7 & Up & $8.42 \mathrm{E}-03$ & 0.64 & hsa04723;hsa04724;hsa04725;hsa04726;hsa04727; \\
& & & & & hsa04728 \\
16840876 & VAMP2 & Up & $1.91 \mathrm{E}-02$ & 0.41 & hsa04721 \\
16713085 & KIF5B & Down & $4.93 \mathrm{E}-02$ & -0.59 & hsa04728 \\
17070634 & RIPK2 & Down & $4.98 \mathrm{E}-02$ & -0.53 & hsa04722 \\
\hline
\end{tabular}

v3.24.10 [10] was used for this purpose.

$$
g_{k} \approx \beta_{0}+\beta_{1} G+\beta_{2} T+\beta_{3} P P+\beta_{4}(T \cdot P P)+\epsilon
$$

A specific contrast matrix was defined to get the relevant expression differences. Given this, estimated coefficients and standard errors were computed from the original model (1). Moderated t-statistics for these contrasts were obtained using an empirical Bayes method. Genes with significant differential expression were selected and ranked based on their resulting adjusted pvalue (5\%). FDR correction for multiple testing was applied. STRING database [11] was used to visualize the protein interactions based on the ranked genes.

\section{Results and discussion}

56 genes were found to be differentially expressed as a response to the acute physical exercise. Among them, a subset of 18 genes were only significant for elite runners group while remain insignificant for active ones, which would suggest that training regime induced a modulation effect in such response. Table 3 shows this list of genes including the KEGG pathways to which they belong. Figure 1 shows an interaction network between the proteins encoded by this subset.

A significant down-regulation is observed in UQCR11, COX7C and COX4I1 gene expression levels taken from cardiac muscle contraction pathway. They are related to mitochondrial respiratory chain function (complexes III and IV specifically). This may indicate mitochondrial impairment due to exercise overload as found in [12] during an experiment with rat myocardium. Further evidence also associates complex IV activity with muscle oxidative capacity for human skeletal muscle [13], in correspondence with our results.

Down-regulation was found for ITPR3 gene, which encodes a receptor for a second messenger that mediates the release of intracellular calcium $\left(\mathrm{Ca}^{2+}\right)$, a key regulator of mitochondrial function. It may reveal an improved muscle damage prevention by avoiding mitochondrial matrix $\mathrm{Ca}^{2+}$ overload, which leads to higher generation rate of reactive oxygen species (ROS) [14].

Neurotrophin intracellular signaling cascades were affected based on up-regulation of IRAK3, PLCG2 and PIK3R2 genes which signals enhance cell survival [3]. A previous study related neurotrophins to stability of cardiac sympathetic innervation and in consequence, an indicator of better heart rate control $[15,16]$.

\section{Conclusions}

Gene expression plasticity due to a specific training regime has been analyzed in terms of cardiac function and neural regulation. A modulated biological response to physical effort has been detected identifying genes related to mitochondrial respiratory chain function and 
neurotrophin signaling pathway. These results generate interesting hypothesis on future experiments with a larger population.

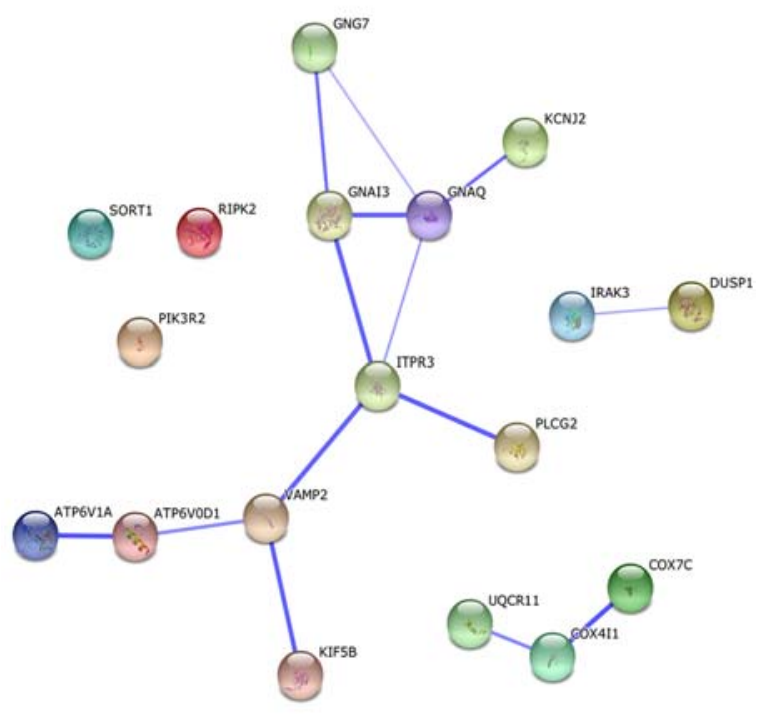

Figure 1. Protein interactions between the 18 ranked genes from Table 3 obtained by STRING database [11]. Thicker lines represent stronger associations under heterogeneous evidence codes.

\section{Acknowledgements}

This work was supported by TEC2014-60337-R grant from the Ministerio de Economía y Competitividad (MINECO). CIBER of Bioengineering, Biomaterials and Nanomedicine is an initiative of ISCIII. Authors are part of the 2014SGR-1063 consolidated research group of the Generalitat de Catalunya, Spain.

\section{References}

[1] Millet GP, Millet GY. Ultramarathon is an outstanding model for the study of adaptive responses to extreme load and stress. BMC Medicine 2012; 10:77.

[2] Melia U, Vallverdu M, Roca E, Brotons D, Irurtia A, Cadefau JA, Caminal P, Perera A. Heart Rate Variability in Ultra-Trail Runners. Computing in Cardiology 2014; 41:997-1000.

[3] Mueller PJ. Exercise training and sympathetic nervous system activity: evidence for physical activity dependent neural plasticity. Clinical and Experimental Pharmacology and Physiology 2007; 34:377-384.

[4] Vance DD, Chen GL, Stoutenberg M, Myerburg RJ, Jacobs K, Nathanson L, Perry A, Seo D, Goldschmidt-Clermont PJ, Rampersaud E. Cardiac Performance, biomarkers and gene expression studies in previously sedentary men participating in half-marathon training. BMC Sports Science, Medicine and Rehabilitation 2014; 6:6.

[5] Timmons JA, Knudsen S, Rankinen T, Koch LG, Sarzynski
M, Jensen T, Keller P, Scheele C, Vollaard NBJ, Nielsen S, Akerström T, MacDougald OA, Jansson E, Greenhaff PL, Tarnopolsky MA, van Loon LJC, Pedersen BK, Sundberg CJ, Wahlestedt C, Britton SL, Bouchard C. Using molecular classification to predict gains in maximal aerobic capacity following endurance exercise training in humans. Journal Applied Physiology 2010; 108:1487-1496.

[6] Kanehisa M, Goto S. KEGG: Kyoto Encyclopedia of Genes and Genomes. Nucleic Acids Research 2000; 28:27-30.

[7] R Core Team (2015). R: A language and environment for statistical computing. $\mathrm{R}$ Foundation for Statistical Computing, Vienna, Austria. URL http://www.Rproject.org/.

[8] Huber W, Carey VJ, Gentleman R, Anders S, Carlson M, Carvalho BS, Bravo HC, Davis S, Gatto L, Girke T, Gottardo R, Hahne F, Hansen KD, Irizarry RA, Lawrence M, Love MI, MacDonald J, Obenchain V, Oles AK, Pages H, Reyes A, Shannon P, Smyth GK, Tenenbaum D, Waldron L, Morgan M. (2015). Orchestrating highthroughput genomic analysis with Bioconductor. Nature Methods 2015; 12:115-121.

[9] Carvalho BS, Irizarry RA. A Framework for Oligonucleotide Microarray Preprocessing. Bioinformatics 2010; 16: 2363-2367.

[10] Ritchie ME, Phipson B, Wu D, Hu Y, Law CW, Shi W, Smyth GK. limma powers differential expression analyses for RNA-sequencing and microarray studies. Nucleic Acids Research 2015; 43(7), e47.

[11] Jensen LJ, Kuhn M, Stark M, Chaffron S, Creevey C, Muller J, Doerks T, Julien P, Roth A, Simonovic M, Bork P, von Mering C. STRING 8 - a global view on problems on proteins and their functional interactions in 630 organisms. Nucleic Acids Research 2009; 37:D412-6.

[12] Kadaja L, Eimre M, Paju K, Roosimaa M, Podramagi T, Kaasik P, Pehme A, Orlova E, Mudist M, Peet N, Piirsoo A, Seene T, Gellerich FN, Seppet EK. Impaired oxidative phosphorylation in overtrained rat myocardium. Experimental \& Clinical Cardiology 2010; 15:e116-127.

[13] Larsen S, Nielsen J, Hansen CH, Nielsen LB, Wibrand F, Stride N, Schroder HD, Boushel R, Helge JW, Dela F, HeyMogensen M. Biomarkers of mitochondrial content in skeletal muscle of healthy young human subjects. Journal of Physiology 2012; 590: 3349-3360.

[14] Brookes PS, Yoon Y, Robotham JL, Anders MW, Sheu SS. Calcium, ATP and ROS: a mitochondrial love-have triangle. American Journal of Physiology - Cell Physiology 2004; 287(4): C817-C833.

[15] Caporali A, Emanueli C. Cardiovascular actions of neurotrophins. Physiological Reviews 2009; 89: 279-308.

[16] Habecker BA, Bilimoria P, Linick C, Gritman K, Lorentz CU, Woodward W, Birren SJ. Regulation of cardiac innervation and function via p75 neurotrophin. Autonomic Neuroscience 2008; 140:40-48.

Address for correspondence.

María Maqueda maria.maqueda@upc.edu Universitat Politècnica de Catalunya (UPC) Facultat de Matemàtiques i Estadística Pau Gargallo 5, 08028 Barcelona, Spain 